\title{
TV Sparsifying MR Image Reconstruction in Compressive Sensing
}

\section{Yonggui Zhu, Xiaolan Yang}

School of Science, Communication University of China, Beijing, China

Email:ygzhu@cuc.edu.cn

Received January $23^{\text {rd }}, 2011$; revised February $18^{\text {th }}, 2011$; accepted February $19^{\text {th }}, 2011$

\begin{abstract}
In this paper, we apply alternating minimization method to sparse image reconstruction in compressed sensing. This approach can exactly reconstruct the MR image from under-sampled $k$-space data, i.e., the partial Fourier data. The convergence analysis of the fast method is also given. Some MR images are employed to test in the numerical experiments, and the results demonstrate that our method is very efficient in MRI reconstruction.
\end{abstract}

Keywords: Compressed Sensing, Magnetic Resonance Image, Total Variation, Image Reconstruction

\section{Introduction}

Compressed Sensing has enormous potentials for scan time reducing significantly in magnetic resonance image(MRI) research community. Compressed Sensing theory was put forward by Candes, Romberg and Tao [1], and D. Donoho [2] in 2006. They pointed out that sparse signals can be reconstructed from a very limited number of samples, provided that the measurements satisfy an incoherence property [3]. For MRI Compressed Sensing, it is possible to reconstruct accurately MR images from under-sampled k-space data, i.e., the partial Fourier data by solving optimization problems.

Suppose $u \in R^{N}$ is a sparse signal, and $\mathrm{K}=\|u\|_{0}$ denotes number of non-zeros in $\mathrm{u}$. Let A be $M \times N(\mathrm{~K}<$ $M \ll N$ ) measurement matrix such that $A u=b$, where $\mathrm{b}$ is an observed data vector. Then to recover $\mathrm{u}$ from $\mathrm{b}$ is equivalent to solve $L_{0}$ - problem:

$$
\min _{u}\left\{\|u\|_{0}, A u=b\right\}
$$

However, problem (1) is provably NP-hard [4] and very difficult to solve from the viewpoint of numerical computation. Thus, it is rexalistic to solve $L_{1}-$ problem:

$$
\min _{u}\left\{\|u\|_{1}, A u=b\right\}
$$

which has also been known to yield sparse solutions under some conditions (see $[5,6]$ for explains). In the case of Compressed Sensing MRI, A is a partial Fourier matrix, i.e., $A=P F, P \in R^{M \times N}$ is consisted of $M \ll N$ rows of the identity matrix, $\mathrm{F}$ is a discrete Fourier matrix. When $b$ is contaminated with noise such as Gaussian noise of variance $\sigma^{2}$, the relaxation form for problem (2) should be given by

$$
\min _{u}\left\{\|u\|_{1}:\|A u-b\|_{2}^{2} \leq \sigma^{2}\right\}
$$

The total variation regularization has been first proposed for image denoising by Rudin, Osher and Fatemi [7]. It is well known that TV regularizier can better recover piecewise smooth signals with preserving sharp edges or boundaries. So TV regularizer is a sparsifying transform operator for piecewise smooth MR images such as brain images. When only TV sparsifying transform is considered, the optimization problem in Compressed Sensing MRI can be written as

$$
\min _{u}\left\{\|u\|_{T V},\|P F u-b\|_{2}^{2} \leq \sigma^{2}\right\}
$$

The unconstrained version of problem (4) is

$$
\min _{u}\|u\|_{T V}+\frac{\mu}{2}\|P F u-b\|_{2}^{2},
$$

where $\mu$ is a positive parameter that determines the trade-off between the fidelity term and the sparsity term, $\|.\|_{2}$ denotes the Euclidean norm. Model (5) was previously mentioned by He etal.[8] and Lustig et al. [9].

In this paper, we focus on the two dimensional MRI Compressed Sensing model (5). All two dimensional images are changed into one dimensional vectors in the context of paper. For square images, let $u \in R^{n^{2}}$ be a 
$n \times n$ image. $\|u\|_{T V}$ in (5) is defined as

$\|u\|_{T V}=\sum_{j, k}\left(\left(\nabla_{1} u_{j, k}\right)^{2}+\left(\nabla_{2} u_{j, k}\right)^{2}\right)^{1 / 2}$. In the two dimen-

sional image practical applications, we only consider the periodic boundary condition of the image. Therefore, the discrete gradient operators $\nabla_{1}$ and $\nabla_{2}$ of $n \times n$ image can be defined by

$$
\begin{aligned}
& \nabla_{1} u_{j, k}=\left\{\begin{array}{lll}
u_{j+1, k}-u_{j, k} & \text { if } & j<n \\
u_{1, k}-u_{n, k} & \text { if } & j=n
\end{array}\right. \\
& \nabla_{2} u_{j, k}=\left\{\begin{array}{lll}
u_{j, k+1}-u_{j, k} & \text { if } & k<n \\
u_{j, 1}-u_{j, n} & \text { if } & k=n
\end{array}\right.
\end{aligned}
$$

$D^{(1)}, D^{(2)} \in R^{n^{2} \times n^{2}}$ denote finite difference matrices corresponding to $\nabla_{1} u_{j, k}$ and $\nabla_{2} u_{j, k}$ respectively. Let $D_{i} u=\left(\left(D^{(1)} u\right)_{i},\left(D^{(2)} u\right)_{i}\right)^{T} \in R^{2} \quad$ where $\left(D^{(l)} u\right)_{i}, l=1,2$ are the $(i=j n+k)$ th entry of $D^{(l)} u, \mathrm{i}$ is corresponding to the $(\mathrm{j}, \mathrm{k})$ th pixel position of the image. The summation $\sum_{i=1}^{n^{2}}\left\|D_{i} u\right\|_{2}$ equals to the discrete total variation of $\mathrm{u}$, i.e. $\|u\|_{T V}$ Thus (5) is changed into

$$
\min _{u} \sum_{i=1}^{n^{2}}\left\|D_{i} u\right\|_{2}+\frac{\mu}{2}\|P F u-b\|_{2}^{2}
$$

Recently, Y. L. Wang et al. [10] have studied the total variation image restoration model:

$$
\min _{u}\|u\|_{T V}+\frac{\mu}{2}\|B u-f\|_{2}^{2}
$$

i.e.

$$
\min _{u} \sum_{i=1}^{n^{2}}\left\|D_{i} u\right\|_{2}+\frac{\mu}{2}\|B u-f\|_{2}^{2},
$$

where $B \in R^{n^{2} \times n^{2}}$ represents a blurring operator. They proposed an alternating minimization method to solve (9). In this paper, we will apply this approach to the Compressed Sensing MRI model (8).

In model (8), $P \in R^{m \times n^{2}}$ is a selection matrix consisting of $m \ll n^{2}$ rows of the identity matrix, $\mathrm{F}$ is a two-dimensional discrete Fourier transform matrix. So $\mathrm{PF}$ serves as a sensing matrix. $\mathrm{b}$ is acquired (by coils in an MRI scanner) and sent to a computer, so b is an observed data containing noise. We will employ quadratic penalty approach [11] to split the model (8) into two sub-minimization problems, and build the fast method to solve the two sub-problems. We will also analyze the convergence of the fast reconstruction method. This paper is organized as follows. In section 2, basic algorithm and optimization is given. Section 3 presents the convergence analysis of the fast reconstruction algorithm.
In section 4, Shepp-Logan Phantom image and some real MR images are employed to do numerical experiments to demonstrate the effectiveness of the fast reconstruction method.

\section{Basic Algorithm and Optimization}

Firstly, we introduce some notations for the sake of convenience. For vectors $\omega_{i} \in R^{n^{2}}$ and finite difference matrices $D^{(i)} \in R^{n^{2} \times n^{2}}, i=1,2$, assume

$w=\left(\omega_{1} ; \omega_{2}\right)=\left(\omega_{1}^{T}, \omega_{2}^{T}\right)^{T}$

and $D=\left(D^{(1)} ; D^{(2)}\right)=\left(D^{(1)^{T}}, D^{(2)^{T}}\right)^{T}$ Let

$w_{i}=\left(\left(\omega_{1}\right)_{i} ;\left(\omega_{2}\right)_{i}\right) \in R^{2}, i=1,2, \cdots \cdots n^{2}$,

$v \in R^{n^{2}}$

By using quadratic penalty approach, we can change (8) into the following problem:

$$
\min _{u, w}\left(\sum_{i=1}^{n^{2}}\left\|w_{i}\right\|_{2}+\frac{\beta}{2} \sum_{i=1}^{n^{2}}\left\|w_{i}-D_{i} u\right\|_{2}^{2}\right)+\frac{\mu}{2}\|P F u-b\|_{2}^{2}
$$

In (11), $w_{i}$ is the approximation of $D_{i} u$. It is well known that the solution of (11) converges to that of (8) as $\beta \rightarrow \infty$. Therefore the solution of (11) for $\beta$ large enough can better approximate to that of (8). If fix alternatively $\mathrm{u}$ and $\mathrm{w}$, we can obtain the following two sub-problems:

$$
\begin{aligned}
& \min _{w} \sum_{i=1}^{n^{2}}\left\|w_{i}\right\|_{2}+\frac{\beta}{2} \sum_{i=1}^{n^{2}}\left\|w_{i}-D_{i} u\right\|_{2}^{2}, \\
& \min _{u} \frac{\beta}{2} \sum_{i=1}^{n^{2}}\left\|w_{i}-D_{i} u\right\|_{2}^{2}+\frac{\mu}{2}\|P F u-b\|_{2}^{2} .
\end{aligned}
$$

Since w-subproblem (12) is separable with respect to $w_{i}$, minimizing (12) is equivalent to solving

$$
\min _{w_{i}}\left\|w_{i}\right\|_{2}+\frac{\beta}{2}\left\|w_{i}-D_{i} u\right\|_{2}^{2}, i=1,2, \cdots, n^{2}
$$

Therefore, we can get its minimizer by two dimensional shrinkage:

$$
w_{i}=s_{w}\left(D_{i} u\right), i=1,2, \cdots, n^{2},
$$

where $s_{w}\left(D_{i} u\right)=\max \left\{\left\|D_{i} u\right\|-\frac{1}{\beta}, 0\right\} \frac{D_{i} u}{\left\|D_{i} u\right\|}$ in which $0 \cdot\left(\frac{0}{0}\right)=0$ is assumed.

For vectors $x, y \in R^{n^{2}}$, define $S_{w}(x, y): R^{2 n^{2}} \rightarrow R^{2 n^{2}}$ by

$$
S_{w}(x, y)=\left(s_{w}\left(z_{1}\right), s_{w}\left(z_{2}\right), \cdots, s_{w}\left(z_{n^{2}}\right)\right)^{T},
$$

where $z_{i}=\left(x_{i}, y_{i}\right)^{T}, i=1,2, \cdots, n^{2}$ 
Rewrite (15) as the following:

$$
w=s_{w}\left(D^{(1)} u, D^{(2)} u\right) .
$$

For u-subproblem (13), the first-order optimality condition is

$$
\beta \sum_{i=1}^{n^{2}} D_{i}^{T} D_{i} u-\beta \sum_{i=1}^{n^{2}} D_{i}^{T} w_{i}+\mu(P F)^{T}(P F u-b)=0
$$

We note that

$$
\begin{gathered}
\sum_{i=1}^{n^{2}} D_{i}^{T} D_{i} u=\left(D^{(1)^{T}} D^{(1)}+D^{(2)^{T}} D^{(2)}\right) u=D^{T} D u \\
\sum_{i=1}^{n^{2}} D_{i}^{T} w_{i}=D^{T} w
\end{gathered}
$$

Thus

$$
\left(\beta D^{T} D+\mu F^{T} P^{T} P F\right) u=\beta D^{T} w+\mu F^{T} P^{T} b .
$$

For l $=1,2$, suppose the Fourier transform of $D^{(l)}$ are $\tilde{D}^{(l)}$. Let

$$
\tilde{D}=\left(\tilde{D}^{(1)} ; \tilde{D}^{(2)}\right)=\left(\tilde{D}^{(1)^{T}}, \tilde{D}^{(2)^{T}}\right)^{T}
$$

Apply the Fourier transform to both sides of (19), we can obtain

$$
\beta \tilde{D}^{T} \tilde{D} F u+\mu P^{T} P F u=\beta \tilde{D}^{T} F w+\mu P^{T} b .
$$

For the periodic boundary condition for u, $D^{(1)^{T}} D^{(1)}$, $D^{(2)^{T}} D^{(2)}$ are all block circulant [12]. Therefore, $\beta D^{T} D$ can be diagonalized by Fourier transform. It is easy to see that $P^{T} P$ is diagonal. Thus $\beta \tilde{D}^{T} \tilde{D}+P^{T} P$ is also a diagonal matrix. Given $w$, we can obtain u by the two steps. The first step is to get $F u$ by solving (20). The second step is to obtain $u$ by applying the inverse fourier transform to $F u$. For the sake of simplicity, let

$$
s_{u}(w)=\min _{u} \frac{\beta}{2} \sum_{i=1}^{n^{2}}\left\|w_{i}-D_{i} u\right\|_{2}^{2}+\frac{\mu}{2}\|P F u-b\|_{2}^{2} .
$$

For a fixed $\beta$, the alternating approximation algorithm for solving (8) can be given as follows:

Algorithm 1 Input $b, P$ and parameters $\beta>0, \mu$ $>0$. Initialize $u=u^{(0)}$

While stopping criterion is not satisfied, Do

Compute

$$
\begin{aligned}
& w^{(k)}=s_{w}\left(D^{(1)} u^{(k-1)}, D^{(2)} u^{(k-1)}\right), \\
& u^{(k)}=s_{u}\left(w^{(k)}\right), k=1,2, \cdots .
\end{aligned}
$$

\section{End Do}

To solve the u-subproblem use only a FFT transform and a inverse FFT transform, and the solution of $w$ subproblem can be obtained by two dimensional shrink- age, so this alternating algorithm is a fast approach. The stopping criterion will be given in the section numerical experiments. The convergence analysis of this algorithm will be discussed in detail in the next section.

\section{The Convergence Analysis and a Continuation Strategy}

In this section, we give the convergence analysis of the alternating algorithm for fixed $\beta>0$. We give the definition of the firmly non-expansive operator before the presentation of non-expansiveness for two dimensional shrinkage.

Definition 3.1. An operator $s: R^{2} \rightarrow R^{2}$ is firmly nonexpansive if it satisfies the following condition:

$$
\|s(x)-s(y)\|_{2}^{2} \leq\|x-y\|_{2}^{2}-\|(I d-s)(x)-(I d-s)(y)\|_{2}^{2}
$$

where Id is identity operator. It is easy to show that a firmly non-expansive operator $s: R^{2} \rightarrow R^{2}$ is non-expansive, i.e., for any $x, y \in R^{2},\|s(x)-s(y)\|_{2} \leq\|x-y\|_{2}$

Lemma 3.1. If $P(\cdot): R^{2} \rightarrow R^{2}$ is the projection onto $\mathrm{B}=\left\{x \in R^{2}\|x\| \leq \frac{1}{\beta}\right\}$, and for $x \in R^{2}$ the two dimensional shrinkage operator $s_{w}: R^{2} \rightarrow R^{2}$ is defined as $s_{w} \triangleq \max \left\{\|x\|_{2}-\frac{1}{\beta}, 0\right\} \frac{x}{\|x\|_{2}}$ where $0 \cdot\left(\frac{0}{0}\right)=0$ is followed, then $s_{w}(x)=x-P(x)$.

Lemma 3.2. For all $x, y \in R^{2}$, it holds that $\left\|s_{w}(x)-s_{w}(y)\right\|_{2}^{2} \leq\|x-y\|_{2}^{2}-\|P(x)-P(y)\|_{2}^{2} \quad$ Furthermore, if $\left\|s_{w}(x)-s_{w}(y)\right\|_{2}=\|x-y\|_{2}$,.then , $S_{w}(x, y): R^{2 n^{2}} \rightarrow R^{2 n^{2}}=x-y$

The proofs of these two lemmas are shown in [10].

By Lemma 3.1 and Lemma 3.2, we can get the following theorem:

Theorem 3.1. For $x \in R^{2}$, if the two dimensional shrinkage operator $S_{w}: R^{2} \rightarrow R^{2}$ is given by $s_{w} \triangleq \max \left\{\|x\|_{2}-\frac{1}{\beta}, 0\right\} \frac{x}{\|x\|_{2}}$ then $S_{w}(\cdot)$ is non-expansive, i.e., for any $x, y \in R^{2}$,

$$
\left\|s_{w}(x)-s_{w}(y)\right\|_{2} \leq\|x-y\|_{2} .
$$

Theorem 3.2. For vectors $x, y \in R^{n^{2}}$, if

$S_{w}(x, y): R^{2 n^{2}} \rightarrow R^{2 n^{2}}$ is defined by

$$
S_{w}(x, y)=\left(s_{w}\left(z_{1}\right), s_{w}\left(z_{2}\right), \cdots, s_{w}\left(z_{n^{2}}\right)\right)^{T}
$$

where $z_{i}=\left(x_{i}, y_{i}\right)^{T}, i=1,2, \cdots, n^{2}$ and $D=\left(D^{(1)} ; D^{(2)}\right)=$ $\left(D^{(1)^{T}}, D^{(2)^{T}}\right)^{T}$, then 
$\left\|S_{w}\left(D^{(1)} x, D^{(2)} x\right)-S_{w}\left(D^{(1)} y, D^{(2)} y\right)\right\|_{2} \leq\|D(x-y)\|_{2}$

Proof. By theorem 3.1 and the definition of $S_{w}$, for any $x, y \in R^{n^{2}}$, we obtain

$$
\begin{aligned}
& \left\|S_{w}\left(D^{(1)} x, D^{(2)} x\right)-S_{w}\left(D^{(1)} y, D^{(2)} y\right)\right\|_{2}^{2} \\
& =\sum_{i=1}^{n^{2}} \| S_{w}\left(\left(\left(D^{(1)} x\right)_{i},\left(D^{(2)} x\right)_{i}\right)^{T}\right) \\
& -S_{w}\left(\left(\left(D^{(1)} y\right)_{i},\left(D^{(2)} y\right)_{i}\right)^{T}\right) \|_{2}^{2} \\
& \leq \sum_{i=1}^{n^{2}}\left\|\left(\left(D^{(1)} x\right)_{i},\left(D^{(2)} x\right)_{i}\right)^{T}-\left(\left(D^{(1)} y\right)_{i},\left(D^{(2)} y\right)_{i}\right)^{T}\right\|_{2}^{2} \\
& =\|D x-D y\|_{2}^{2}
\end{aligned}
$$

since $D=\left(D^{(1)} ; D^{(2)}\right)=\left(D^{(1)^{T}}, D^{(2)^{T}}\right)^{T}$

This completes the proof.

Let $M=\beta D^{T} D+\mu F^{T} P^{T} P F$, since $\beta>0, \mu>0, \mathrm{M}$ is nonsingular. According to (19), we have

$$
\begin{aligned}
& u^{(k)}=S_{u}\left(w^{(k)}\right) \\
& =M^{-1}\left(\beta D^{T} w^{(k)}+\mu F^{T} P^{T} b\right) \\
& =M^{-1}\left(\beta D^{T} S_{w}\left(D^{(1)} u^{(k-1)}, D^{(2)} u^{(k-1)}\right)+\mu F^{T} P^{T} b\right) \\
& =\beta M^{-1} D^{T} S_{w}\left(D^{(1)} u^{(k-1)}, D^{(2)} u^{(k-1)}\right)+\mu M^{-1} F^{T} P^{T} b
\end{aligned}
$$

\section{Assume}

$$
\begin{aligned}
\mathrm{T}\left(u^{(k-1)}\right) & =\beta M^{-1} D^{T} S_{w}\left(D^{(1)} u^{(k-1)}, D^{(2)} u^{(k-1)}\right), \\
& +\mu M^{-1} F^{T} P^{T} b
\end{aligned}
$$

Then

$$
u^{(k)}=\mathrm{T}\left(u^{(k-1)}\right)
$$

Next we show that the operator $\mathrm{T}$ is non-expansive.

Theorem 3.3. For $\mu$ large enough in (8), the operator $\mathrm{T}$ in (24) is non-expansive, i.e., for $x, y \in R^{n^{2}}$, it holds that

$$
\|T(x)-T(y)\|_{2} \leq\|x-y\|_{2}
$$

Proof.

$$
\begin{aligned}
& \|T(x)-T(y)\|_{2} \\
& =\left\|\beta M^{-1} D^{T}\left(S_{w}\left(D^{(1)} x, D^{(2)} x\right)-S_{w}\left(D^{(1)} y, D^{(2)} y\right)\right)\right\|_{2} . \\
& \leq \beta\left\|M^{-1} D^{T}\left(S_{w}\left(D^{(1)} x, D^{(2)} x\right)-S_{w}\left(D^{(1)} y, D^{(2)} y\right)\right)\right\|_{2}
\end{aligned}
$$

Using (23) in Theorem 3.2, we have

$$
\begin{aligned}
& \left\|M^{-1} D^{T}\left(S_{w}\left(D^{(1)} x, D^{(2)} x\right)-S_{w}\left(D^{(1)} y, D^{(2)} y\right)\right)\right\|_{2} \\
& \leq\left\|M^{-1} D^{T} D(x-y)\right\|_{2}
\end{aligned}
$$

Hence

$$
\begin{aligned}
& \|T(x)-T(y)\|_{2} \leq \beta\left\|M^{-1} D^{T} D(x-y)\right\|_{2} \\
& \leq \beta\left\|M^{-1} D^{T} D\right\|\|x-y\|_{2} \\
& \leq\left(\beta\left\|D^{T} D\right\|\right)\left\|M^{-1}\right\|\|x-y\|_{2}
\end{aligned}
$$

where $\|\cdot\|$ is matrix-norm.

Since $M=\beta D^{T} D+\mu F^{T} P^{T} P F$, if $\mu$ is large enough such that $\left(\beta\left\|D^{T} D\right\|\right)\left\|M^{-1}\right\| \leq 1$, then $\|T(x)-T(y)\|_{2}$ $\leq\|x-y\|_{2}$

This completes the non-expansiveness of the operator $\mathrm{T}(\cdot)$.

Theorem 3.4. For any initial value $u^{(0)} \in \mathbb{R}^{n^{2}}$, assume $\left\{u^{(k)}\right\}$ be generated by (25), then $\mathrm{T}$ is asymptotically regular, i.e.,

$$
\lim _{k \rightarrow \infty}\left\|u^{(k+1)}-u^{(k)}\right\|_{2}=\lim _{k \rightarrow \infty}\left\|T^{k+1}\left(u^{(0)}\right)-T^{k}\left(u^{(0)}\right)\right\|_{2}=0
$$

Proof. Since

$$
w^{(k)}=S_{w}\left(D^{(1)} u^{(k-1)}, D^{(2)} u^{(k-1)}\right)
$$

by (19), we have

$$
\begin{aligned}
& \left(\beta D^{T} D+\mu F^{T} P^{T} P F\right) u^{(k+1)} \\
= & \beta D^{T} S_{w}\left(D^{(1)} u^{(k)}, D^{(2)} u^{(k)}\right)+\mu F^{T} P^{T} b \\
( & \left.\beta D^{T} D+\mu F^{T} P^{T} P F\right) u^{(k)} \\
= & \beta D^{T} S_{w}\left(D^{(1)} u^{(k-1)}, D^{(2)} u^{(k-1)}\right)+\mu F^{T} P^{T} b
\end{aligned} .
$$

(26) minus (27) is

$$
\begin{aligned}
& \left(\beta D^{T} D+\mu F^{T} P^{T} P F\right)\left(u^{(k+1)}-u^{(k)}\right) \\
& =\beta D^{T}\left(S_{w}\left(D^{(1)} u^{(k)}, D^{(2)} u^{(k)}\right)-S_{w}\left(D^{(1)} u^{(k-1)}, D^{(2)} u^{(k-1)}\right)\right)
\end{aligned}
$$

That is

$$
\begin{aligned}
u^{(k+1)}-u^{(k)} & \\
= & M^{-1} \beta D^{T}\left(S_{w}\left(D^{(1)} u^{(k)}, D^{(2)} u^{(k)}\right)\right. \\
& \left.-S_{w}\left(D^{(1)} u^{(k-1)}, D^{(2)} u^{(k-1)}\right)\right)
\end{aligned}
$$

Therefore

$$
\begin{aligned}
& \left\|u^{(k+1)}-u^{(k)}\right\|_{2} \leq\left\|M^{-1} \beta D^{T}\right\| \\
& \left\|\left(S_{w}\left(D^{(1)} u^{(k)}, D^{(2)} u^{(k)}\right)-S_{w}\left(D^{(1)} u^{(k-1)}, D^{(2)} u^{(k-1)}\right)\right)\right\|_{2}
\end{aligned}
$$


Using non-expansiveness of $S_{w}$, we obtain

$$
\left\|u^{(k+1)}-u^{(k)}\right\|_{2} \leq\left\|M^{-1} \beta D^{T}\right\|\left\|D\left(u^{(k)}-u^{(k-1)}\right)\right\|_{2}
$$

That is

$$
\left\|u^{(k+1)}-u^{(k)}\right\|_{2} \leq \rho\left\|u^{(k)}-u^{(k-1)}\right\|_{2}
$$

where $\rho=\left\|M^{-1} \beta D^{T}\right\|\|D\|$

When $\mu$ is large enough such that $\rho<1$, then we have $\left\|u_{\text {So }}^{(k+1)}-u^{(k)}\right\|_{2} \leq \rho^{k}\left\|u^{(1)}-u^{(0)}\right\|_{2}$

$$
\lim _{k \rightarrow \infty}\left\|u^{(k+1)}-u^{(k)}\right\|_{2}=0
$$

This completes the proof.

We note that the objective function in (11) is convex, bounded below, and coercive, thus (11) has at least one minimizer $\left(u^{*}, w^{*}\right)$, and must satisfy

$$
\begin{gathered}
u^{*}=S_{u}\left(w^{*}\right) \\
w^{*}=S_{w}\left(D^{(1)} u^{*}, D^{(2)} u^{*}\right)
\end{gathered}
$$

So $u^{*}$ is a fixed point of $\mathrm{T}$.

According to the Opial theorem [13], the squence $\left\{u^{(k)}\right\}$ converges to a fixed point of $\mathrm{T}$.

The algorithm 1 given in the section 2 can be significantly accelerated by employing a continuation scheme introduced by Y. Wang [10]. That means penalty parameters $\beta$ vary with $\mathrm{k}$, starting from initial small values and increase them gradually. In the implementation of the algorithm, firstly for small fixed $\beta$ apply the algorithm to solve (11) up to the stopping criterion. Next the obtained solution is used as new starting point of applying the algorithm to (11) for the next $\beta$. Go on like this until the the given $\beta_{\max }$ is obtained. Such a continuation scheme is also called path-following technique which is widely used in the penalty methods [14-17]. This accelerating convergence result is also verified by our numerical experiments. Now we present the fast reconstruction method (FRM) for MR images with TV sparsity, which will be be used in the numerical experiments.

Algorithm 2 Input $\mathrm{b}, \mathrm{P}, \mu>0, \beta_{0}>0$ and $\beta_{\max }>\beta_{0}$. Initialize $u=u^{(0)}$

While $\beta<\beta_{\max }$, Do

1) For fixed $\beta$, run Algorithm 1 until the stopping criterion is satisfied.

2) Update $\beta \leftarrow 2 * \beta$.

\section{End Do}

\section{Numerical Experiments}

In this section, we present the performance of fast reconstruction method(FRM) for MR images with TV sparsity.
We compare our method with Two-step iterative shrinkage/ thresholding algorithm(TwIST) [18], which can be used to solve (8). The general model solved by TwIST is

$$
\min _{u} \frac{1}{2}\|A u-b\|_{2}^{2}+\tau \Phi(u),
$$

where $\Phi$ is a regularization function such that the solution of the denoising problem

$$
\Psi_{\tau}(v)=\min _{u} \frac{1}{2}\|v-u\|_{2}^{2}+\tau \Phi(u),
$$

is known. If set $\Phi(u)=\sum_{i=1}^{n^{2}}\left\|D_{i} u\right\|_{2}, \tau=\frac{1}{\mu}, A=P F$, then (28) is identical to (8). That is the reason that we choose TwIST method to compare with our method.

Here we give the iteration framework of TwIST to solve (28) in brief. TwIST is a two-step version of iterative shrinkage/thresholding(IST) [19] algorithm. The iteration formula of TwIST is as follows:

$$
\begin{gathered}
\Psi_{\tau}\left(v_{k}\right)=\min _{u} \tau \Phi(u)+\frac{1}{2}\left\|v_{k}-u\right\|_{2}^{2}, \\
u_{k+1}=(1-\alpha) u_{k-1}+(\alpha-\beta) u_{k}+\beta \Psi_{\tau}\left(v_{k}\right),
\end{gathered}
$$

where $\alpha, \beta>0 \quad v_{k}=u_{k}+A^{T}\left(b-A u_{k}\right)$, In the latest software, TwIST_v1, $\alpha$ and $\beta$ are set as

$$
\begin{gathered}
\alpha=\hat{\alpha} \equiv \hat{\rho}^{2}+1, \\
\beta=\hat{\beta} \equiv 2 \hat{\alpha} /\left(\bar{\varsigma}_{m}+\varsigma_{1}\right)
\end{gathered}
$$

In which, the detail explain of parameters $\varsigma_{1}, \bar{\varsigma}_{m}$ and $\hat{\rho}$ can be seen in [18]. In TwIST_ v1, denoising problem (30) is solved iteratively by Chambolle's algorithm [20]. Signal to noise ratio (SNR) and relative error (ReErr) are used to measure the quality of the reconstructed images. The definitions of SNR and ReErr are given as follows:

$$
\begin{gathered}
S N R=10 \log 10\left(\frac{\left\|u_{0}\right\|_{2}}{\left\|u_{0}-u\right\|_{2}}\right), \\
\operatorname{Re} E r r=\frac{\left\|u_{0}-u\right\|_{2}^{2}}{\left\|u_{0}\right\|_{2}^{2}},
\end{gathered}
$$

where $u$ and $u_{0}$ are the reconstructed image and original image, respectively. All experiments were done in MATLAB on a laptop with Intel Core Duo P8400 processor and 2 GB of memory.

The $256 \times 256$ Shepp-Logan phantom image is sampled with 22 views in frequency space, for which there are 6136 Fourier coefficients measured, i.e., sample ratio is $9.36 \%$. Figure 1 shows 22 radial lines in frequency space. 


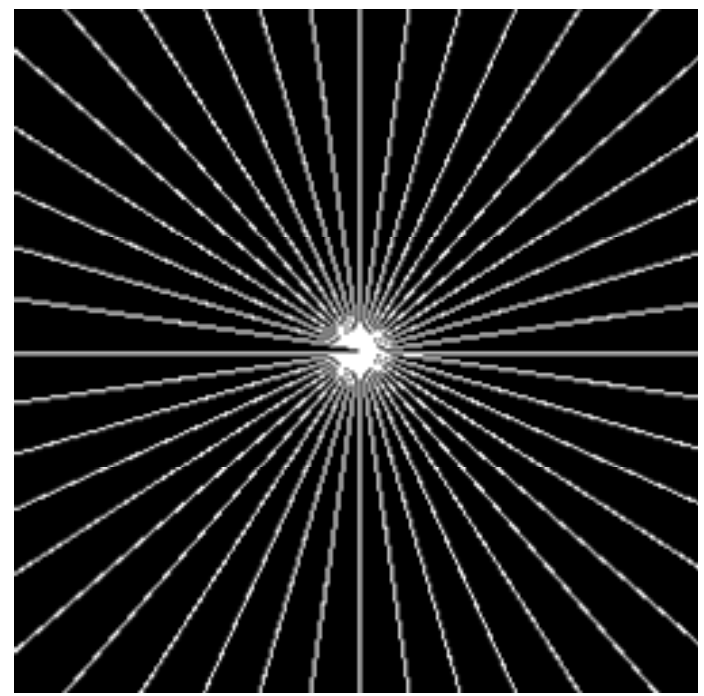

Figure 1. Sampling domain with 22 views in frequency space.

In the following tests, we assume the mean value and standard deviation for the additive Gaussian noise are 0 and 0.01, respectively. When using FRMto do numerical experiments, we initialize $\beta=2^{5}$ and final $\beta_{\max }$ equals to $2^{10}$. Let $\mu=1000$, and set initial image $u^{(0)}$ to zero. The stopping criterion is the relative difference between the successive iterate of the reconstructed image satisfy the following:

$$
\frac{\left\|u^{(k)}-u^{(k-1)}\right\|_{2}}{\left\|u^{(k)}\right\|_{2}}<10^{-4}
$$

According to the above mentioned parameters and the stopping criterion, we use our algorithm to do Phantom image reconstruction experiment. Figure 2(a) is PheppLogan phantom original image. The reconstructed image is showed in Figure 2(b). The SNR, ReErr and CPU time(seconds) are $31.3687 \mathrm{~dB}, 0.0270$ and 19.4375 , respectively.

Now we apply TwIST method to do Phantom image reconstruction experiment. We choose the monotonic variant of TwIST in TwIST_v1, which stops when the relative change in the objective function falls below Tolerance $=1 \mathrm{e}-4$. Parameters $\alpha$ and $\beta$ are determined by using (32) and (33). The parameter $\varsigma_{1}$ is equal to 1e-3 that is recommended by the TwIST_v1 documentation. Set $\tau=1 \mathrm{e}-3$, which is corresponding to $\mu=1000$.

If we use TwIST to do the reconstruction test for the Logan Phantom image with same sampling ratios. The result is presented in Figure 2(c). Its SNR, ReErr and CPU time(seconds) are $29.0010 \mathrm{~dB}, 0.0355$ and 55.5938, respectively. From all the two reconstructed images, it is clear that our method have better qualities than TwIST. In addition, from the values of SNR, ReErr and CPU time for restructed image, our method increases 11 the SNR by $2.3677 \mathrm{~dB}$ and reduce the ReErr and CPU time 0.0085 and 35.1563 seconds more than TwIST.

When we choose some views 44, 66, 88, the sampling ratios for Phantom image are $18.76 \%$, 26.85\%, 34.97\%. The reconstructed results are showed in Figure $\mathbf{3}$ and Figuure 4 by the two methods.

The following table presents the values of SNR, ReErr and CPU time of reconstructions under different views by FRM and TwIST.

From Table 1, we can see that the SNR obtained using FRM is higher than that obtained using TwIST under the same sampling ratios or views. Figure 5(a), (b) and (c) give SNRs, the relative errors and CPU time, respectively, of reconstructed images by FRM (red curves) and TwIST (green curves) from the observed data at a sequence of different sampling ratios.

Now we look three different $256 \times 256$ MR brain images, which are shown in Figure 6 (a), (b) and (c), respectively.

We choose 66 views sampling for the three MR images, in which the sampling ratio is equal to $26.85 \%$.

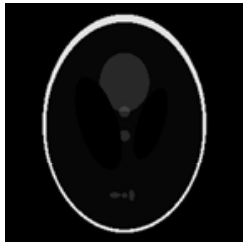

(a)

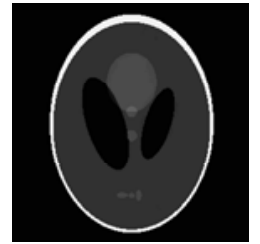

(b)

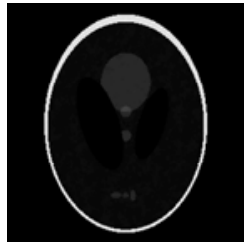

(c)
Figure 2. (a) Original image. (b) Reconstruction by FRM. (c) Reconstruction by TwIST.

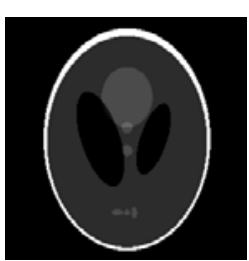

(a)

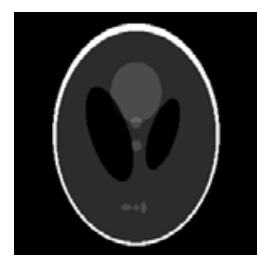

(b)

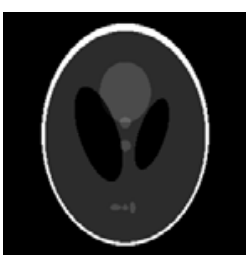

(c)
Figure 3. Reconstruction by FRM (a) 44 views. (b) 66 views. (c) 88 views.

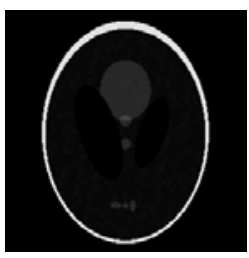

(a)

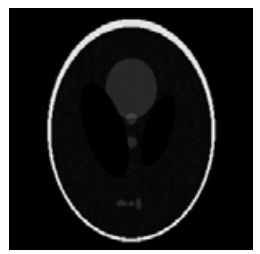

(b)

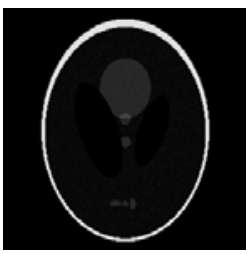

(c)
Figure 4. Reconstruction by TwIST (a) 44 views. (b) 66 views. (c) 88 views. 
Table 1. SNR, ReErr, CPU of reconstructions under different views or sampling ratios by two methods.

\begin{tabular}{lllll}
\hline Views/Samp.ratio & Method & SNR & ReErr & CPU \\
\hline $44 / 18.76 \%$ & FRM & 40.6877 & 0.0092 & 7.1875 \\
& TwIST & 30.7696 & 0.0289 & 26.4219 \\
$66 / 26.85 \%$ & FRM & 44.8714 & 0.0057 & 4.9063 \\
& TwIST & 30.8412 & 0.0287 & 20.2031 \\
$88 / 34.97 \%$ & FRM & 47.8810 & 0.0040 & 3.6719 \\
& TwIST & 30.8412 & 0.0286 & 17.7969 \\
\hline
\end{tabular}

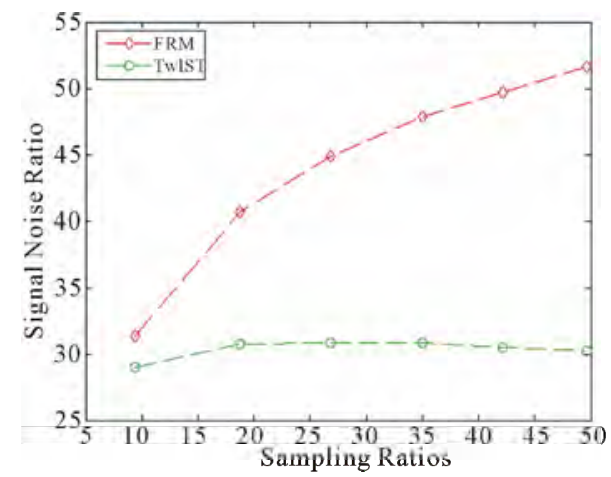

(a)

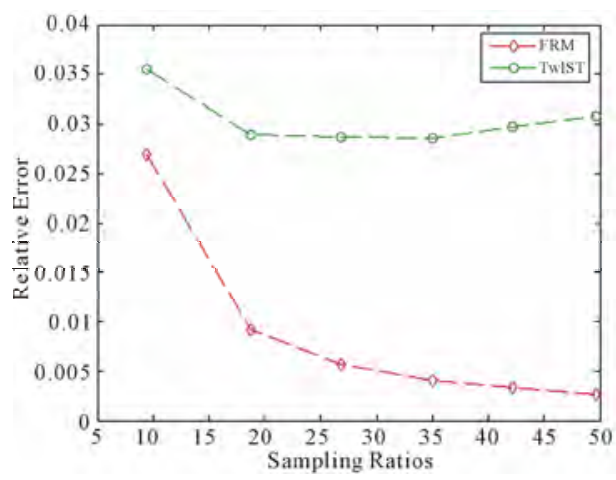

(b)

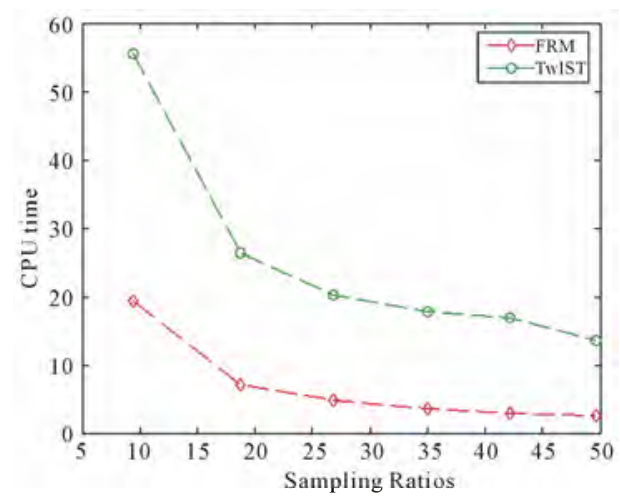

(c)

Figure 5. (a) SNRs. (b) Relative Errors. (c) CPU time.
When use FRM and TwIST to do reconstruction experiments for the three MR images, all parameters in the two methods are still same as the above mentioned. The results reconstructed by FRM and TwIST are presented in Figure 7. and Figure 8., respectively.

The SNRs, the relative errors and CPU time of the reconstructed images by FRM and TwIST are given in the Table 2.

From the quality of reconstructed images and the values of SNR, ReErr and CPU time, we can see that FRM method is better than TwIST method.

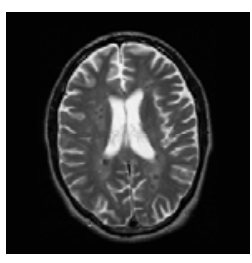

(a)

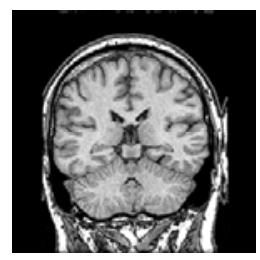

(b)

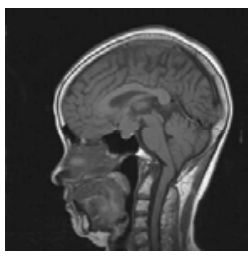

(c)
Figure 6. Original image. (a) Brain 1. (b) Brain 2. (c) Brain 3.

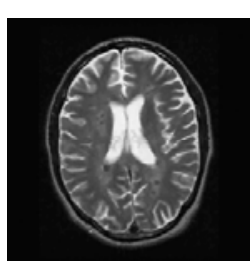

(a)

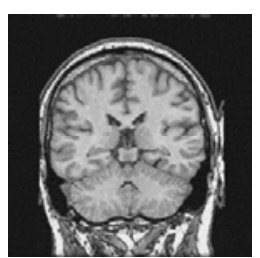

(b)

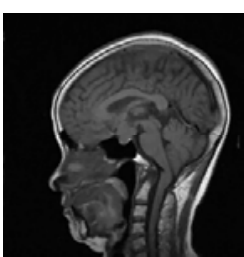

(c)
Figure 7. Reconstructions by FRM (a) Brain 1. (b) Brain 2. (c) Brain 3.

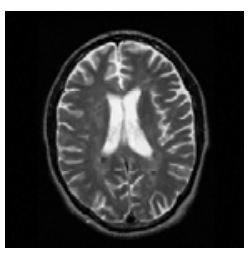

(a)

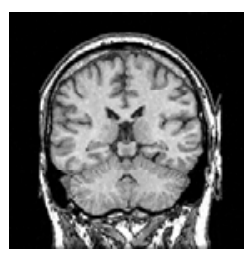

(b)

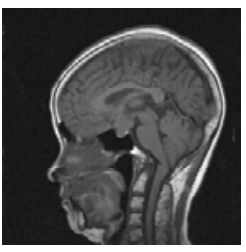

(c)
Figure 8. Reconstructions by TwIST (a) Brain 1. (b) Brain 2. (c) Brain 3.

Table 2. SNR, ReErr, CPU time and sampling ratios for reconstructed results by FMR and TwIST.

\begin{tabular}{llllll}
\hline Image & Method & SNR & ReErr & CPU & Samp.ratio \\
\hline Brain & FRM & 26.1288 & 0.0494 & 6.9063 & $26.85 \%$ \\
1 & TwIST & 24.7365 & 0.0580 & 20.8438 & $26.85 \%$ \\
\multirow{2}{*}{ Brain } & FRM & 21.9353 & 0.0800 & 9.1719 & $26.85 \%$ \\
2 & TwIST & 21.1494 & 0.0876 & 20.9375 & $26.85 \%$ \\
Brain & FRM & 27.6916 & 0.0412 & 6.4531 & $26.85 \%$ \\
3 & TwIST & 26.1599 & 0.0492 & 20.5938 & $26.85 \%$ \\
\hline
\end{tabular}




\section{Conclusion}

We have developed a fast reconstruction method for compressed sensing MRI that is called FRM. The convergence of the reconstruction method has been analyzed. The algorithm has been accelerated by continuation on penalty parameters. FRM is compared with TwIST, a state-of-the-art method. We use the two methods to reconstruct Phantom image and some real MR images from a partial of observed data. The results of numerical experiments on the MR images demonstrate that FRM can achieve much higher performance in terms of SNRs, the relative errors and CPU time than TwIST. We believe that our method can be applied in the area of rapid MR imaging.

\section{Acknowledgements}

This work was supported by the Key Project of Chinese Ministry of Education(No. 109030), 382 Training Programme of CUC(G08382316) and Science Research Project of CUC(XNL1003). The authors would like to thank the referees for very useful suggestions here.

\section{REFERENCES}

[1] E. J. Candes, J. Romberg, and T. Tao, "Robust uncertainty principles: Exact signal reconstruction from highly incomplete frequency information," IEEE Trans. Inf. Theory, Vol. 52, 2006, pp. 489-509.

[2] D. L. Donoho, “Compressed sensing,” IEEE Trans. Inf. Theory, Vol. 52, 2006, pp. 1289-1306.

[3] E. Candes and J. Romberg, "Sparsity and incoherence in compressive sampling,” Inverse Problems, Vol. 23, 2007, pp. 969-985.

[4] B. K. Natarajan, "Sparse approximate solutions to linear systems,” SIAM J. Comput., Vol. 24, 1995, pp.227-234.

[5] D. Donoho and X. Huo, "Uncertainty principles and ideal atomic decompositions,” IEEE Trans. Inf.Theory, Vol. 47, 2001, pp. 2845-2862.

[6] J. J. Fuchs, "On sparse representations in arbitrary redundant bases,” IEEE Trans. Inf. Theory, Vol. 50, 2004, pp. 1341-1344.

[7] L. Rudin, S. Osher and E. Fatemi, "Nonlinear total variation based noise removal algorithms,” Physica D, Vol. 60, 1992, pp. 259-268.
[8] L. He, T. C. Chang, S. Osher, T. Fang and P. Speier, "MR image reconstruction by using the iterative refinement method and nonlinear inverse scale space methods," UCLA CAM Report, 2006,06-35.

[9] M. Lustig, D. Donoho and J. Pauly, "Sparse MRI: The application of compressed sensing for rapid MR imaging,” Magnetic Resonance in Medicine, Vol. 58,2007,pp. 1182-1195.

[10] Y. Wang, J. Yang, W. Yin and Y. Zhang, “A alternating minimization algorithm for total variation image reconstruction,” SIAM J. Imag. Sci., Vol. 1, 2008,pp. 248-272.

[11] R. Courant, "Variational methods for the solution of problems with equilibrium and vibration," Bull.Amer. Math. Soc., Vol. 49, 1943, pp. 1-23.

[12] M. K. Ng, R. H. Chan and W. C. Tang, “A fast algorithm for deblurring models with Neumann boundary conditions,” SIAM J. Sci. Comput., Vol. 21, pp. 851-866, 1999.

[13] Z. Opal, "Weak convergence of the sequence of successive approximations for nonexpansive mappings," Bull. Amer. Math. Soc., Vol. 73, 1967, pp. 591-597.

[14] J. Yang, W. Yin and Y.Wang, “A Fast Algorithm for Edge-Preserving Variational Multichannel Image Restoration,” SIAM J. Sci. Comput., Vol. 2, 2009, pp. 569-592.

[15] J. Yang, Y. Zhang and W. Yin, “A Fast Alternating Direction Method for TVL1-L2 Signal Reconstruction From Partial Fourier Data," IEEE Journal of Selected Topics in Signal Processing, Vol. 4, 2010, pp.288-297.

[16] E. T. Hale, W. Yin and Y. Zhang, “A Fixed-Point Continuation for 11-regularization with Application to Compressed Sensing," Rice University CAAM Technical Report TR07-07, 2007, pp. 1-45.

[17] E. T. Hale, W. Yin and Y. Zhang, "Fixed-Point Continuation for 11-Minimization: Methodology and Convergence,” SIAM J. Sci. Comput., Vol. 2, 2009, pp. 569-592.

[18] J. Bioucas-Dias and M. Figueiredo, "A new TwIST: Two-step iterative thresholding algorithm for image restoration,” IEEE Trans. Imag. Process., Vol. 16, 2007, pp. 2992-3004.

[19] I. Daubechies, M. Defriese and C. De Mol, "An iterative thresholding algorithm for linear inverse problems with a sparsity constraint,” Commun. Pure Appl. Math., Vol. 57, 2004, pp. 1413-1457.

[20] A. Chambolle, “An algorithm for total variation minimization and applications,” J. Math. Imaging Vision, Vol. 20, 2004, pp. 89-97. 